\title{
$k$-fold mixing lifts to weakly mixing isometric extensions
}

\author{
DANIEL J. RUDOLPH \\ Department of Mathematics, University of Maryland, College Park, Md. 20742, USA
}

(Received 12 August 1984)

Abstract. If $\hat{T}$ is a weakly mixing isometric extension of a finite measure preserving, $k$-fold mixing map $T$, then $\hat{T}$ must also be $k$-fold mixing.

We here complete a collection of results each of which reads 'If $T$ is $[\cdots]$ and $\hat{T}$ is a weakly mixing isometric extension of $T$, then $\hat{T}$ is $[\cdots]$,' where $[\cdots]$ can be [weakly mixing], [ $k$-fold mixing], [K] or [Bernoulli]. The first is trivial. Each of the others has a distinctly different proof (see [1] for $K$ and [2] for Bernoulli). Here we will prove the $k$-fold mixing case.

Let $(T, X, \mathscr{F}, \mu)$ be an ergodic finite measure preserving transformation of a non-atomic Lebesgue probability space. Let $Y$ be a compact metric space with a transitive group $G$ of isometries. By a $G$-cocycle $f(x, n)$ over $T$ we mean a measurable $\operatorname{map} f: \Omega \times \mathbb{Z} \rightarrow G$ so that

$$
f\left(x, n_{1}+n_{2}\right)=f\left(T^{n_{1}}(x), n_{2}\right) \circ f\left(x, n_{1}\right)
$$

i.e.

$$
f(x, n)=\prod_{i=0}^{n-1} f\left(T^{i}(x), 1\right) .
$$

We will abbreviate $f(\omega, 1)=f(\omega)$, the generating function of the cocycle.

As $G$ is transitive on $Y, Y$ is isometric to $G / H, H$ an isotropy subgroup of some point $y_{0}$, and Haar measure on $G$ projects to a $G$-invariant normalized measure $\nu$ on $Y$.

On the probability space $(X \times Y, \mathscr{F} \times \mathscr{G}, \mu \times \nu)$ we can define a measure preserving action $\hat{T}$ by

$$
\hat{T}^{n}(x, y)=\left(T^{n}(x), f(x, n)(y)\right) .
$$

We call this the 'f-extension' of $T$, and generically an 'isometric extension' of $T$.

THEOREM 1. If $T$ is $k$-fold mixing, i.e. for any measurable sets $A_{0}, A_{1}, \ldots, A_{k-1}$,

$$
\lim _{n_{1}, n_{i+1}-n_{i} \rightarrow \infty} \mu\left(A_{0} \cap T^{n_{1}}\left(A_{1}\right) \cdots \cap T^{n_{k-1}}\left(A_{k-1}\right)\right)=\mu\left(A_{0}\right) \mu\left(A_{1}\right) \cdots \mu\left(A_{k}\right),
$$

and $\hat{T}$ is a weakly mixing isometric extension of $T$, then $\hat{T}$ is also $k$-fold mixing.

Proof. It is enough to verify for functions

$$
g_{i}(x, y)=\chi_{A_{i}}(x) \bar{g}_{i}(y),
$$


where $\chi_{A_{i}}$ is the characteristic function of $A_{i} \in \mathscr{F}$ and $\bar{g}_{i}$ is continuous and $\leq 1$, that

$$
\begin{aligned}
\lim _{n_{1}, n_{i+1}-n_{i} \rightarrow \infty} & \left(\int g_{0}(x, y) g_{1}\left(\hat{T}^{n_{1}}(x, y)\right) \cdots g_{k-1}\left(\hat{T}^{n_{k-1}}(x, y)\right) d \mu \times \nu\right) \\
= & \prod_{i=0}^{k-1} \int g_{i}(x, y) d \mu \times \nu,
\end{aligned}
$$

as such functions generate an $L^{1}$ dense algebra.

Assume $\bar{g}_{1}, \ldots, \bar{g}_{k-1}$ are fixed continuous functions, and $\delta(\varepsilon)$ a uniform modulus of continuity for all $(k-1)$ of them.

As $\hat{T}$ is weakly mixing, the $k$-fold product $\hat{T} \times \hat{T} \times \cdots \times \hat{T}$ acting on $\left((X \times Y)^{k},(\mathscr{F} \times \mathscr{G})^{k},(\mu \times \nu)^{k}\right)$ is ergodic. Thus for $(\mu \times \nu)^{k}$-a.e. point $\left(\left(x_{0}, y_{0}\right), \ldots,\left(x_{k-1}, y_{k-1}\right)\right)$,

$$
\lim _{n \rightarrow \infty} \frac{1}{n} \sum_{i=0}^{n-1}\left(\prod_{j=0}^{k-1} g_{j}\left(\hat{T}^{i}\left(x_{j}, y_{j}\right)\right)\right)=\prod_{i=0}^{k-1} \int g_{i}(x, y) d \mu \times \nu .
$$

Fix an $\varepsilon>0$ and select $\bar{y}_{1}, \ldots, \bar{y}_{s}$, a $\delta\left(\varepsilon / 2^{k+3}\right)$ dense subset of $Y$, and partition $Y$ into sets $B_{i}, y_{i} \in B_{i}$, of diameter less than $\delta\left(\varepsilon / 2^{k+3}\right)$. Let $0<\alpha=\min \left(\nu\left(B_{i}\right)\right)$.

Thus for all points $\left(x_{0}, \ldots, x_{k-1}\right) \in X^{k}$ and $\left(y_{0}, \ldots, y_{k-1}\right),\left(y_{0}^{\prime}, \ldots, y_{k-1}^{\prime}\right) \in Y^{k}$ if $y_{k}$ and $y_{k}^{\prime}$ are in the same $B_{i(k)}$,

$$
\frac{1}{N} \sum_{i=0}^{N-1}\left(\prod_{j=0}^{k-1} g_{j}\left(\hat{T}^{i}\left(x_{j}, y_{j}^{\prime}\right)\right)\right)=\frac{1}{N} \sum_{i=0}^{N-1}\left(\prod_{j=0}^{k-1} g_{j}\left(\hat{T}^{i}\left(x_{j}, y_{j}\right)\right)\right) \pm \frac{\varepsilon}{8}
$$

Select $N$ so large that for $(\mu \times \nu)^{k}$ all but $\varepsilon \alpha^{k} / 4$ of the points $\left(\left(x_{0}, y_{0}\right), \ldots,\left(x_{k-1}, y_{k-1}\right)\right)$,

$$
\frac{1}{N} \sum_{i=0}^{N-1}\left(\prod_{j=0}^{k-1} g_{j}\left(\hat{T}^{i}\left(x_{j}, y_{j}\right)\right)\right)=\prod_{i=0}^{k-1} \int g_{i}(x, y) d \mu \times \nu \pm \frac{\varepsilon}{8}
$$

It now follows that for $\mu^{k}$ all but $\varepsilon / 4$ of the points $\left(x_{0}, \ldots, x_{k-1}\right)$, for all $\left(y_{0}, y_{1}, \ldots, y_{k-1}\right)$,

$$
\frac{1}{N} \sum_{i=0}^{N-1}\left(\prod_{j=0}^{k-1} g_{j}\left(\hat{T}^{i}\left(x_{j}, y_{j}\right)\right)\right)=\prod_{j=0}^{k-1} \int g_{j}(x, y) d \mu \times \nu \pm \frac{\varepsilon}{4},
$$

as the existence of one point $\left(y_{0}, \ldots, y_{k-1}\right)$ not satisfying this error bound implies a set of measure at least $\alpha^{k}$ not satisfying the earlier error bound for a given $\left(x_{0}, \ldots, x_{k-1}\right)$. Partition $X$ into subsets $C_{1}, \ldots, C_{p}$ so that if $x_{1}, x_{2} \in C_{j}$ and $n=$ $1, \ldots, N$ then

$$
\left|f\left(x_{1}, n\right)-f\left(x_{2}, n\right)\right|<\delta\left(\varepsilon / 2^{k+2}\right),
$$

and

each $A_{i}$ is a union of $C_{j}$ 's.

Thus if $x_{j}, x_{j}^{\prime} \in C_{k(j)}$ for $j=0, \ldots, k-1$ then

$$
\frac{1}{N} \sum_{i=0}^{N-1}\left(\prod_{j=0}^{k-1} g_{j}\left(\hat{T}^{i}\left(x_{j}, y_{j}\right)\right)\right)=\frac{1}{N} \sum_{i=0}^{N-1}\left(\prod_{j=0}^{k-1} g_{j}\left(\hat{T}^{i}\left(x_{j}^{\prime}, y_{j}\right)\right)\right) \pm \frac{\varepsilon}{4} .
$$


Now as $T$ is $k$-fold mixing, we can select $M$ so large that if $n_{1}, n_{i+1}-n_{i}>M$, then for any $C_{j(0)}, \ldots, C_{j(k-1)}$,

$$
\mu\left(C_{j(0)} \cap T^{-n_{1}}\left(C_{j(1)}\right) \cap \cdots\left(T^{-n_{k-1}}\left(C_{j(k-1)}\right)\right)=\prod_{i=0}^{k-1} \mu\left(C_{i(j)}\right)\left(1 \pm \frac{\varepsilon}{4}\right) .\right.
$$

Fix such a choice of $n_{1}, \ldots, n_{k-1}$ and construct an invertible measure preserving map $\phi:(X, \mathscr{F}, \mu) \rightarrow\left(X^{k}, \mathscr{F}^{k}, \mu^{k}\right)$ so that for all but $\varepsilon / 4$ of the $x \in X$, if $\left(x, T^{n_{1}}(x), \ldots, T^{n_{k-1}}(x)\right) \in C_{i(0)} \times C_{i(1)} \times \cdots \times C_{i(k-1)}$ then $\phi(x) \in\left(C_{i(0)} \times C_{i(1)} \times \cdots\right.$ $\left.\times C_{i(k-1)}\right),\left(\phi(x)=\left(\phi(x)_{1}, \phi(x)_{2}, \ldots, \phi(x)_{k}\right)\right)$.

Now

$$
\begin{aligned}
\int \prod_{j=0}^{k-1}\left(g_{j}\left(\hat{T}^{n_{j}}(x, y)\right)\right) d \mu \times \nu & =\int \frac{1}{N} \sum_{i=0}^{N-1}\left(\prod_{j=0}^{k-1} g_{j}\left(\hat{T}^{n_{j}+i}(x, y)\right)\right) d \mu \times \nu \\
& =\int \frac{1}{N} \sum_{i=0}^{N-1}\left(\prod_{j=0}^{k-1} g_{j}\left(\hat{T}^{i}\left(\phi(x)_{j}, f\left(x, n_{j}\right)(y)\right)\right)\right) d \mu \times \nu \pm \frac{\varepsilon}{4}
\end{aligned}
$$

But for $\mu^{k}$ all but $\varepsilon / 4$ of the $\phi(x)$, for all $y$,

$$
\frac{1}{N} \sum_{i=0}^{N-1} \prod_{j=0}^{k-1} g_{j}\left(\hat{T}^{j}\left(\phi(x)_{j}, f\left(x, n_{j}\right)(y)\right)=\prod_{j=0}^{k-1} \int g_{j}(x, y) d \mu \times \nu \pm \frac{\varepsilon}{2} .\right.
$$

Hence if $n_{1}, n_{i+1}-n_{i}>M$,

$$
\int \prod_{j=0}^{k-1}\left(g_{j}\left(\hat{T}^{n_{j}}(x, y)\right)\right) d \mu \times \nu=\prod_{j=0}^{k-1} \int g_{j}(x, y) d \mu \times \nu \pm \varepsilon,
$$

completing the result.

\section{REFERENCES}

[1] W. Parry. Ergodic properties of affine transformations and flows on nilmanifolds. Amer. J. of Math. 91 (1969), 757-771.

[2] D. Rudolph. Classifying the isometric extensions of a Bernoulli shift. J. d'Analyse Math. 34 (1978), 36-60. 